\title{
Stochastic Convergence in Regional Economic Activity
}

\author{
Fariba Hashemi \\ Swiss Federal Institute of Technology, Lausanne, Switzerland \\ E-mail: Fariba.Hashemi@epfl.ch \\ Received August 11, 2011; revised September 21, 2011; accepted October 5, 2011
}

\begin{abstract}
A stochastic model is presented, based on a double process of temporal drift and random disturbance, to fit the evolution of cross-country distribution of income and economic activity. Instead of assuming a steady state as is standard practice, a long run stationary equilibrium distribution is hypothesized, around which economic activity fluctuates. An empirical application comparing dynamics of growth in Asia and Europe tests the validity of the proposed method. In particular, results point out that the distribution of income and economic activity is approaching a long run equilibrium at a faster rate in the case of Asia, and that the dispersion of the distribution is shrinking over time above all in the case of Europe. Main implications are supportive of the convergence hypothesis, and suggest that diffusion may be a potential technique for the analysis of growth dynamics.
\end{abstract}

Keywords: Dynamics of Growth, Stochastic Processes

\section{Introduction}

The study is motivated by the observation that early leaders in industrial revolution like the UK and the Netherlands needed over 50 years for income to double from $\$ 2000$ to $\$ 4000$, but countries that reached $\$ 2000$ after 1950 needed 10 - 20 years [1]. It would not be unreasonable to consider that the shortening of time required for doubling per capita income may be attributed to a change in the velocity of factor mobility. Consistent with this observation, we hypothesize that income fluctuates around some long run stationary equilibrium according to a temporal drift and random disturbance. A drift-diffusion model is proposed, to express income adjustment process with noise, where dynamics of income rely on two counteracting forces: 1) a mean-reversion process along time, driven by mobility of factors of production and 2) a diffusion process across regions, driven by search and learning and trial and error. The present paper is one of a rather small group of evolutionary studies which eschews simulation in favour of analytical derivations.

\section{Theoretical Framework}

Debates on growth theory have contrasted the convergence predictions of the neoclassical growth models of Swan [2] and Solow [3] with predictions of potential non-convergence from endogenous technological pro- gress of Romer [4] and Aghion et al. [5]. In his classic Contribution to the Theory of Economic Growth [3], Solow proposed that we study economic growth by assuming a standard neoclassical production function with decreasing returns to capital. In Solow's world, there are two inputs, capital and labor, which are paid their marginal products. Countries may differ in technology, resource endowments, geography and institutions. In standard neoclassical growth theory with diminishing returns, a country's growth rate will be inversely related to its initial income. If all economies are assumed to have the same steady state, in the absence of shocks, countries at different stages of economic development are predicted to experience absolute convergence. It has been argued that much of the cross-country differences in economic outcomes can be traced to differing determinants of the steady state in the Solow growth model. If, more realistically, economies are assumed to have different steady states contingent on differences in parameters such as accumulation of human and physical capital and population growth, then neoclassical theory only predicts conditional convergence [6].

In particular, since the works of Baumol [7], Barro [8] and Mankiw et al. [9], the existence of convergence in economic outcomes within groups of similar countries has been largely recognized. What is less known, however, is the dynamics in question. The present study helps fill this gap, by providing a novel methodology to summarize 
growth dynamics. A model is proposed to describe the fluctuations over time in the density of cross-sectional distribution of income and economic activity. It is hypothesized that economic activity fluctuates around its long run stationary equilibrium according to a temporal drift and random disturbance. These flows follow simple stochastic laws that can be described with a few parameters; parameters which can be estimated from historical data with some accuracy.

\section{Model}

Consider a region consisting of a constant number of countries with different levels of income and output ${ }^{1}$. The set of incomes forms a distribution which evolves over time. Consider the basic conservation law, with flux interpreted as the number of countries entering and number of countries exiting an income/output interval. Assume that flux is made of two different parts: a mean reversion process - call it drift, and a random process - call it diffusion. The counteracting forces of drift and diffusion result in a long-run stationary equilibrium distribution of economic activity. The equilibrium is a result of tension between counteracting forces of convergence and divergence.

More precisely, for the drift spread, it is assumed that there exists some equilibrium distribution of economic activity with a certain mean and variance, towards which the ensemble of countries gravitates, and the process is governed by a velocity of convergence. Convergence is a result of adjustment of capital-labor ratios to common steady-state levels starting from different initial values [1]. This adjustment is driven by diminishing returns [2,3]. For the diffusion spread, a search and learning process generates randomness in the system [10-13]. Bottlenecks in the flow of labor and capital and random effects cause a spread of income/output from high density towards lower density. Noise is generated by diffusion of knowledge and learning, and limited by the presence of obstacles in the form of trade barriers and the like.

In order to interpret and quantify this relationship, consider a classical linear stochastic differential equation in which the flux of probability consists of a drift and some diffusion. The history of a country's economic activity is governed by an ordinary differential equation

$$
\mathrm{d} X / \mathrm{d} t=g(X, t)
$$

where $g(x, t)$ is the drift. Letting $X(x, t)$ be the solution such that $X(x, 0)=x$, we obtain:

$$
\mathrm{d} X(x, t) / \mathrm{d} t=g(X(x, t), t),
$$

\footnotetext{
${ }^{1}$ In this study, "income" and "output" will be used interchangeably to
} represent economic activity.

$$
X(x, 0)=x
$$

Assume that $x>0$ and that the solution $X(x, t)$ remains positive (see Appendix A).

From the point of view of diffusion processes, consider a stochastic differential equation of the Ito type:

$$
\begin{gathered}
\mathrm{d} X(x, t)=\varepsilon \mathrm{d} W(t)+g(X(x, t)) \mathrm{d} t, \\
X(x, 0)=x
\end{gathered}
$$

where $\varepsilon$ is a small positive constant, and $g$ is the drift. $W$ is a standard Wiener process (see Appendix B).

Consider the form $g(x)=\lambda(x-u)$ where $u>0$, and $\lambda>0 . \lambda$ denotes the adjustment rate, which for simplicity is assumed constant. $u$ denotes the mean of the stationary equilibrium distribution. In this case, each point moves toward the position $u$, but never reaches it. In general, one can study a Markov process generated by a matrix of transitions from one level of income/output to another, where the Markov process can be treated as income/output diffusion. Then one can apply the general Fokker-Planck equation to describe evolution in time of economic activity. Hence, assuming that income/output behaves like a stochastic process and that it is continuous and Markovian, we consider the most natural candidate; a classical linear stochastic differential equation driven by a standard Wiener process ${ }^{2}$. Our picture of world development is thus one where convergence is counterbalanced by divergence. Convergence in the context of the present model would mean collapsing of the cross-section distribution. Divergence would mean that the cross-section distribution replicates itself because for example it happens to be the stationary distribution for many independent and identically-distributed country outputs.

\section{Empirical Analysis}

\subsection{Data}

We use data on GDP per capita for nine Asian economies between the years of 1980 to 2007 . The countries are: China, Hong Kong, Indonesia, Japan, Malaysia, South Korea, Singapore, Taiwan and Thailand. Figure 1 illustrates the evolution of the distribution of average GDP per capita. We observe a steady growth with the East Asian Financial crisis of 1997 standing out. Table 1 reports the descriptive of log GDP per capita for this population.

For comparison, consider GDP per capita for 24 countries in the European Union: Austria, Belgium, Bulgaria, Cyprus, Czech Republic, Denmark, Estonia, Finland,

${ }^{2}$ The process evolves according to an Ornstein-Uhlenbeck. This type of model has been widely used in Biomathematics [15,16]. Ref [17-19] develops and provides a full analysis of this model albeit in a different context. 
Table 1. Descriptive of GDP Asia.

\begin{tabular}{cccccc}
\hline & $\mathrm{N}$ & Years & Minimum & Maximum & Mean \\
\hline All data & 252 & $1980-2007$ & 5.530 & 10.814 & 8.686 \\
Used for fit & 171 & $1989-2007$ & 6.419 & 10.814 & 9.021 \\
\hline
\end{tabular}

Evolution of GDP (Asia)

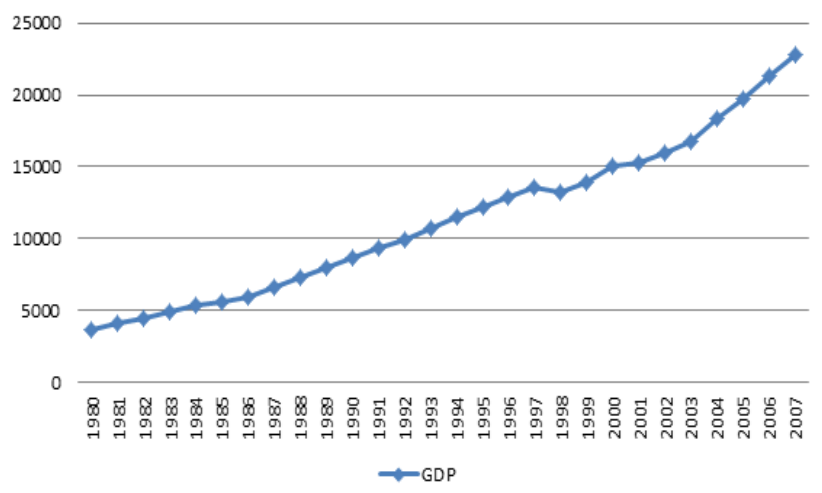

Figure 1. Evolution of GDP Asia.

France, Germany, Greece, Hungary, Iceland, Italy, Latvia, Lithuania, Luxembourg, Netherlands, Poland, Slovakia, Slovenia, Spain, Sweden, and United Kingdom. Data were recorded from 1991 to 2007. Figure 2 illustrates the evolution of the distribution of average GDP per capita. Table 2 reports the descriptive of $\log$ GDP per capita for this population. All data has been collected from Datastream.

\subsection{Estimation}

The expectation of the distribution representing the time development of the differential equation expressing the growth dynamics is:

$$
u_{t}=u\left(1-e^{-\lambda t}\right)+u_{0} e^{-\lambda t}
$$

and the variance of the distribution:

$$
\sigma_{t}^{2}=\sigma_{0}^{2} e^{-2 \lambda t}+\frac{\varepsilon}{\lambda}\left(1-e^{-2 \lambda t}\right)
$$

The model has been applied to log GDP per capita distribution for the two populations as a function of time, using non-linear least squares estimation ${ }^{3}$. A two-step ${ }^{3}$ The expression representing the time-development of the distribution is:

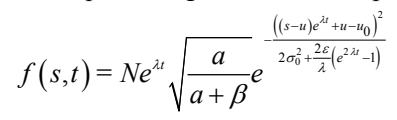

where $N$ is the normalization constant, $a=\frac{\sigma_{0}^{2}}{2}$ and

$$
\beta=\frac{\varepsilon}{2 \lambda}\left(e^{2 \lambda}-1\right)
$$

GDP Evolution (EU)

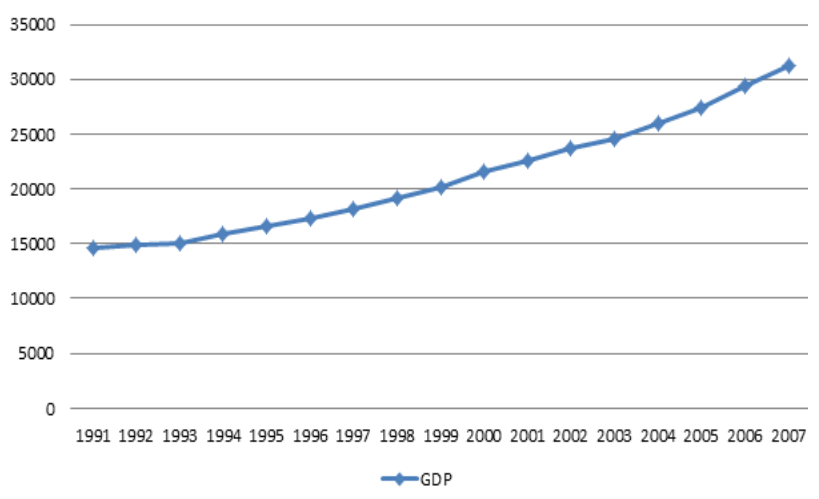

Figure 2. Evolution of GDP EU.

procedure has been employed to estimate the model parameters, where $u_{0}$ denotes the initial mean of the distribution, and $u$ denotes where the mean is heading. $\sigma_{0}$ represents the initial standard deviation, $\varepsilon$ represents the diffusion parameter, and $\lambda$ represents the velocity of income/output convergence.

Tables 3 and 4 report estimates for the five model parameters, along with the standard errors and t-values for Asian and European populations respectively (in logs).

Figures 3 and 4 illustrate the evolution of real versus fitted mean and variance of the distributions for Asia and Europe respectively.

As expected, the mean of the distribution is clearly evolving for both European and Asian populations, corresponding to our theoretical predictions. The variance of the distribution is likewise evolving for both populations. Results point out that the distributions are approaching a long-run equilibrium (at a faster rate in the case of our European economies). One might speculate that this pressure towards reduction of disparities across European countries may be attributed to the establishment of the Maastricht Treaty in 1993. The diffusive limit suggests that the distribution variance will tend toward a constant and concentrated around a mean $u$, which is larger for our Asian population than for our European one.

By considerations of analytical tractability, the model developed in this paper is simplified. An extension which would significantly enrich the analysis would be to include exogenous control variables that shift the conditional mean (e.g., business cycle effects). We also 
Table 2. Descriptive of GDP Europe.

\begin{tabular}{ccccccc}
\hline & N & Years & Minimum & Maximum & Mean & Std. Deviation \\
\hline All data & 685 & $1976-2007$ & 8.123 & 11.290 & 9.596 & 0.578 \\
Used for fit (mu) & 685 & $1976-2007$ & 8.123 & 11.290 & 9.596 & 0.578 \\
Used for fit (sigma) & 200 & $2000-2007$ & 8.732 & 11.290 & 10.047 & 0.488 \\
\hline
\end{tabular}

Table 3. Parameter estimates for Asia.

\begin{tabular}{cccc}
\hline Parameter & Value & Std Error & t-value \\
\hline$\lambda$ & 0.014 & 0.011 & 1.221 \\
$u$ & 13.393 & 3.578 & 3.743 \\
$u_{0}$ & 7.781 & 0.125 & 62.250 \\
$\sigma_{o}^{2}$ & 2.251 & 0.084 & 26.680 \\
$\varepsilon$ & 0.010 & 0.009 & 1.143 \\
\hline
\end{tabular}

Table 4. Parameter estimates for Europe.

\begin{tabular}{cccc}
\hline Parameter & Value & Std Error & t-value \\
\hline$\lambda$ & 0.017 & 0.009 & 1.843 \\
$u$ & 11.913 & 1.287 & 9.254 \\
$u_{0}$ & 8.815 & 0.046 & 190.004 \\
$\sigma_{o}^{2}$ & 1.130 & 0.350 & 3.222 \\
$\varepsilon$ & -0.003 & 0.002 & -1.323 \\
\hline
\end{tabular}

observe that the rate of approach to the final equilibrium as well as the relaxation time in our model only depend on $\lambda$ characterizing the drift term. This feature is entirely inherent to the linearity of the dynamics considered, and turns out to be a limitation in the modeling capability offered by Ornstein-Uhlenbeck. For nonlinear drifts, this feature does not occur anymore and the noise strength strongly affects the transient behavior of the probability density.

\section{Conclusions}

Over the past two centuries, the interplay of geography and policies has produced disparities in economic outcomes. Given the emergence of a global system, parts of the developing world are now narrowing the income gap with richer nations [20]; we witness a tendency towards convergence between nations at different levels of economic development. Monitoring this momentous development requires appropriate techniques for quantification
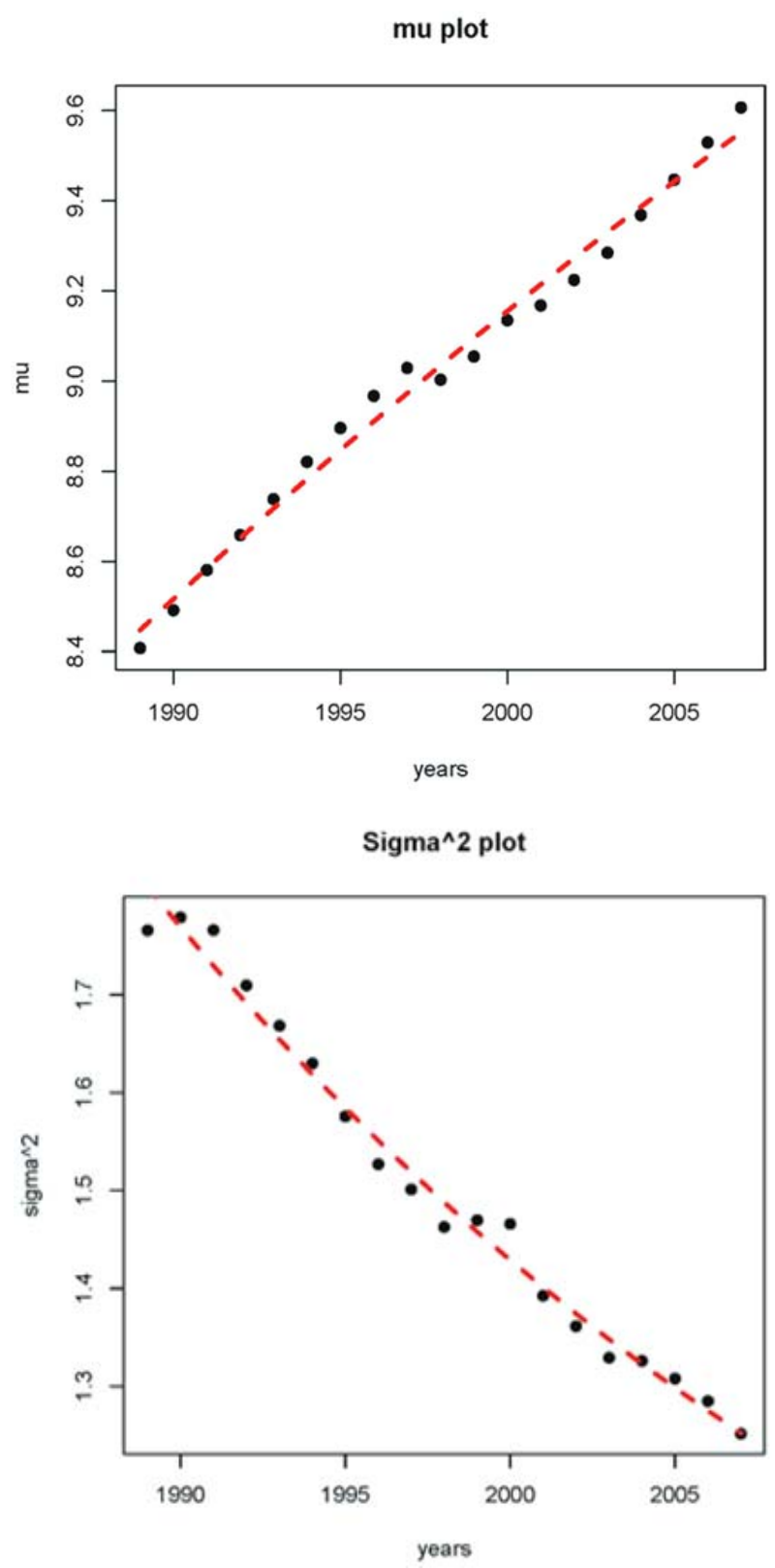

Figure 3. Asia.

and interpretation. This paper proposes one such technique. The results suggest that diffusion is a potential method to monitor regional growth dynamics. 

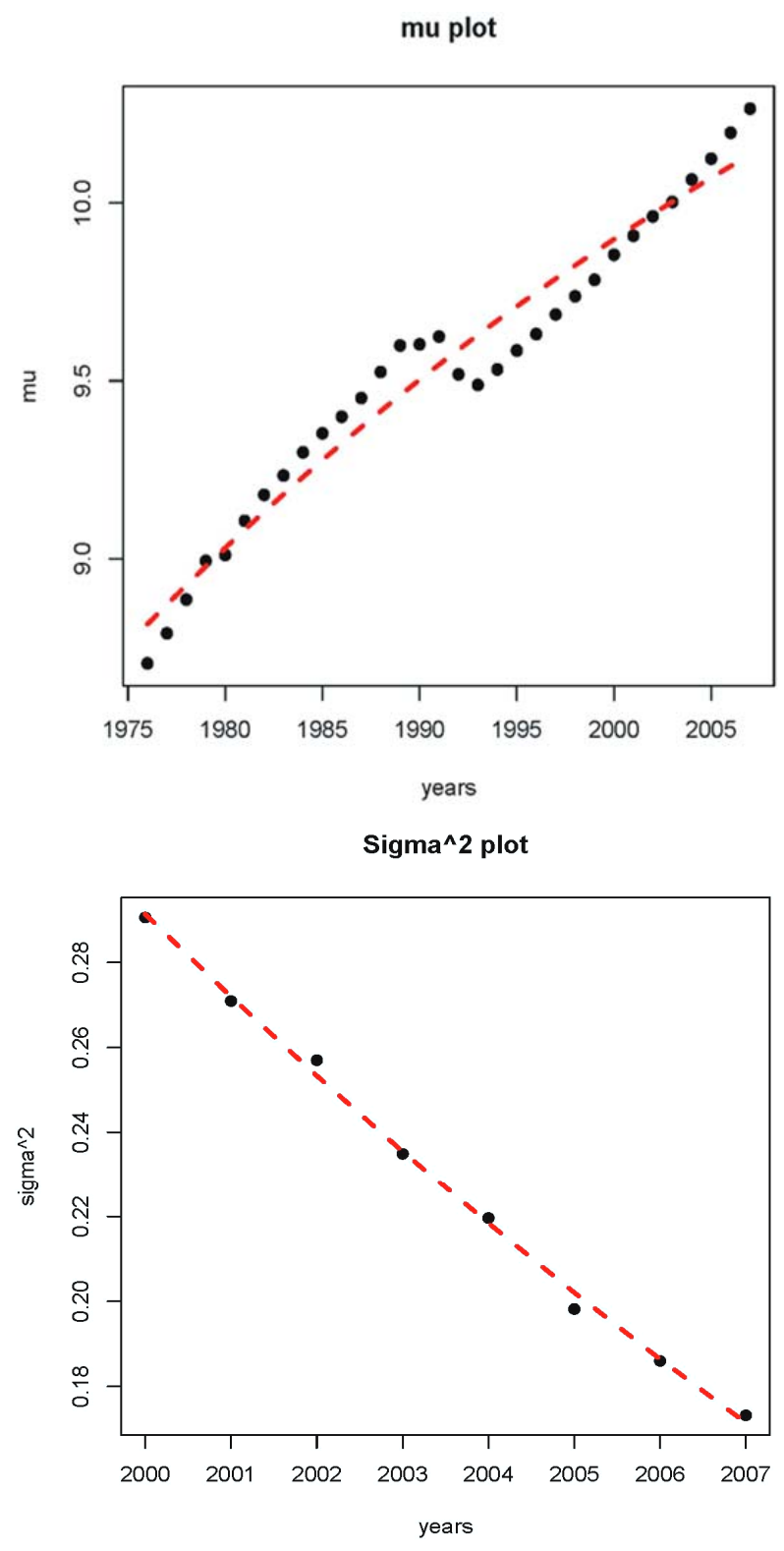

Figure 4. Europe.

\section{Acknowledgments}

Part of this paper has been built on previous work of the author [21]. Moreover, the author is grateful to Mark Hannay for assistance on the estimation section of this paper.

\section{References}

[1] R. Lucas, "Trade and the Diffusion of the Industrial Revolution," American Economic Journal: Macroeconomics, Vol. 1, No. 1, 2009, pp. 1-25.

[2] T. Swan, "Economic Growth and Capital Accumulation,"
Economic Record, Vol. 32, No. 2, 1956, pp. 334-361. doi:10.1111/j.1475-4932.1956.tb00434.X

[3] R. Solow, "A Contribution to the Theory of Economic Growth," Quarterly Journal of Economics, Vol. 70, No. 1, 1956, pp. 65-94. doi:10.2307/1884513

[4] P. Romer, "Endogenous Technological Change," The Journal of Political Economy, Vol. 98, No. 5, 1990, pp. S71-S102. doi:10.1086/261725

[5] P. Aghion and P. Howitt, "Endogenous Growth Theory," MIT Press, Cambridge, 1998.

[6] R. Barro and X. Sala-i-Martin, "Technological Diffusion, Convergence, and Growth," Journal of Economic Growth, Vol. 2, No. 1, 1997, p. 126. doi:10.1023/A:1009746629269

[7] W. Baumol, "Productivity Growth, Convergence, and Welfare: What the Long-Run Data Show," The American Economic Review, Vol. 76, No. 5, 1986, pp. 1072-1085

[8] R. Barro, "Economic Growth in a Cross Section of Countries," Quarterly Journal of Economics, Vol. 106, No. 2, 1991, pp. 407-443. doi:10.2307/2937943

[9] G. Mankiw, D. Romer and D. Weil, "A Contribution to the Empirics of Economic Growth," Quarterly Journal of Economics, Vol. 107, No. 2, 1992, pp. 407-437. doi: $10.2307 / 2118477$

[10] D. Levine and S. Modica, "Anti-Malthus: Evolution, Population, and the Maximization of Free Resources," WUSTL Working Paper, 2011.

[11] D. Levine, "Neuroeconomics," International Review of Economics, Vol. 58, No. 3, 2011, pp. 287-305. doi:10.1007/s12232-011-0128-7

[12] J. Hirshleifer, "Economics from a Biological Viewpoint," Journal of Law \& Economics, Vol. 20, No. 1, 1977, pp. 1-52. doi: $10.1086 / 466891$

[13] J. Hirshleifer, "Competition, Cooperation, and Conflict in Economics and Biology," American Economic Review, Vol. 68, No. 2, 1978, pp. 238-243.

[14] Freidlin, "Annals of Mathematics Study," Princeton University Press, Princeton, 1985.

[15] M.-O. Hongler, H. Soner and L. Streit, "Stochastic Control for a Class of Random Evolution Models," Applied Mathematics and Optimization, Vol. 49, No. 2, 2004, pp. 113-121.

[16] O. Besson and G. de Montmollin, "Space-Time Integrated Least Squares: A Time-Marching Approach," International Journal for Numerical Methods in Fluids, Vol. 44, No. 5, 2004, pp. 525-543. doi:10.1002/fld.655

[17] F. Hashemi, "A Dynamic Model of Size Distribution of Firms Applied to U.S. Biotechnology and Trucking Industries," Small Business Economics, Vol. 21, No. 1, 2003, pp. 27-36. doi:10.1023/A:1024433203253

[18] F. Hashemi, "An Evolutionary Model of the Size Distribution of Firms," Journal of Evolutionary Economics, Vol. 10, No. 5, 2000, pp. 507-521. doi:10.1007/s001910000048

[19] F. Hashemi, "A Dynamic Model for the Cross-sectional 
Distribution of Unemployment Rates," Labour, Vol. 16, No. 1, 2002, pp. 89-102.

[20] J. Sachs, "Nature, Nurture and Growth," The Economist, 12 June 1997. http://www.economist.com/node/91003
[21] F. Hashemi, "East Asian Economic Growth-An Evolutionary Perspective," Journal of Service Science and Management, Vol. 4, No. 3, 2011, pp. 280-283. doi:10.4236/jssm.2011.43033

\section{Appendix A}

This is a degenerate diffusion with no Brownian "noise". Thus the position at time $t$ of income/output starting at $x$ does not have a probability density in the ordinary sense but is deterministic. It is important that a differential equation such as (1) defines a flow on the whole interval $(0, \infty)$. In other words, a set in $(0, \infty)$, for example an interval $(a, b)$, is transformed into another set, the interval $(X(a, t), X(b, t))$, at time $t$. Assuming $g(x, t)$ is a "reasonable" function, the paths from two distinct points, namely $t \rightarrow X(a, t)$ and $t \rightarrow X(b, t)$ will never meet, so an interval remains an interval under this flow. The flow transforms any initial measure on the interval $(0, \infty)$ into a different measure. Suppose the initial measure is given by a density function $f_{0}(x) \geq 0$, where we can normalize by taking $\int_{0}^{\infty} f_{0}(x) \mathrm{d} x=1$. Then the transformed measure attaches to the interval $(a, b)$ the measure $\int_{\alpha}^{\beta} f(x) \mathrm{d} x$, where $\alpha$ and $\beta$ (which depend on $t$ ) are the inverse images of $a$ and $b: X_{t}(\beta)=b, X_{t}(\alpha)=a$. Assuming that $g$ is not very peculiar and thus the transformed measure also has a density, call the transformed density $f_{t}(y)$. One can interpret $f_{t}(y)$ as the mass density at time $t$; alternatively, if we think of $f_{0}(x)$ as the initial probability density for a single country income/ output, then $f_{t}(x)$ is the probability density at time $t$. For the mass density interpretation, we may think of $\int a b f_{t}(y) \mathrm{d} y$ as the fraction of all country incomes that are in the interval $(a, b)$ at time $t$. Our picture of the world thus follows the lines of the derivation used for Kolmogorov's forward differential equation. Taking the probability interpretation, with the initial density $f_{0}(x)$, let $Y_{t}$ be the randomized X-process, using $f_{0}$ for the density of the initial point $x$. Let $w(y)$ be a smooth function vanishing off some finite subinterval of $(0, \infty)$. The expected value $\mathrm{E}\left(w\left(Y_{t}\right)\right)$ can be expressed as:

$$
\int_{0}^{\infty} w(y)\{\partial f(y, t) / \partial t+\partial[g(y) f(y, t)] / \partial y\} \mathrm{d} y=0
$$

Since this expression is 0 for all functions $w(y)$ of the type described above, we can conclude that the bracket \{\} is identically 0 .

\section{Appendix B}

Under mild conditions on $g$, Equation (2) is known to have a unique solution. Moreover, for each $t>0$ and each $x, X(x, t)$ does have a probability density 
$f(x, y, t)$, and $f(x, y, t)$ satisfies Kolmogorov's forward equation:

$$
\begin{aligned}
\partial f(x, y, t) / \partial t= & (1 / 2) \varepsilon \partial^{2} f(x, y, t) / \partial y^{2} \\
& -\partial[g(y, t) f(x, y, t)] / \partial y
\end{aligned}
$$

where $x$ is fixed, indicating the starting point. $f(x, y, 0)$ is a "Dirac" function, with all the mass concentrated at $x$, and so is not a density in the ordinary sense. For $t>0$, one has Prob $\{\mathrm{a} \leq X(x, t) \leq b\}=\int_{a}^{b} f(x, y, t) \mathrm{d} y$. We may again suppose $x$ is initially random. Multiplying this equation by $f_{0}(x)$, integrating over $(0, \infty)$ and letting

$$
f^{*}(y, t)=\int_{-\infty}^{\infty} f(x, y, t) f_{0}(x) \mathrm{d} x,
$$

we get:

$$
\begin{aligned}
\partial f^{*}(y, t) / \partial t= & (1 / 2) \varepsilon \partial^{2} f^{*}(y, t) / \partial y^{2} \\
& -\partial\left[g(y, t) f^{*}(y, t)\right] / \partial y
\end{aligned}
$$

To show that the solution of this equation with $\varepsilon=0$ is the limit of the solution as $\varepsilon \rightarrow 0$, see Ref. [14, Chapter 4]. 